\title{
Tumor Findings Vendor Name
}

National Cancer Institute

\section{Source}

National Cancer Institute. Tumor Findings Vendor Name. NCI Thesaurus. Code C119933.

The literal identifier of the vendor or laboratory that performs a tumor findings assessment. 